\title{
The Use of Dried Blood Spot Samples in Screening Drugs of Abuse
}

\section{Rizwana Quraishi, Raka Jain, Atul Ambekar}

National Drug Dependence Treatment Centre, Department of Psychiatry, All India Institute of Medical Sciences, New Delhi, India. Email: rizwanaquraishi@gmail.com

Received January $24^{\text {th }}, 2013$; revised February $27^{\text {th }}, 2013$; accepted March $30^{\text {th }}, 2013$

Copyright (C) 2013 Rizwana Quraishi et al. This is an open access article distributed under the Creative Commons Attribution License, which permits unrestricted use, distribution, and reproduction in any medium, provided the original work is properly cited.

\begin{abstract}
The present article will provide an overview of use of dried blood sampling method for analysis of drug of abuse. Relatively short half life and instability in blood, calls for alternative sampling method for determination of drugs of abuse. Dried blood spot (DBS) method has many advantages over the conventional sampling methods. The available method for DBS sample collection, storage and transport is described here. The techniques involved in and the factors that may influence the accuracy and reproducibility of the DBS methods for determination of drugs of abuse are presented. The DBS sampling has the potential to be a useful technique to detect drugs of abuse. The use of DBS for any drug should be judged against the potential error involved with the method.
\end{abstract}

Keywords: Dried Blood Spots; Drugs of Abuse; Screening; Review

\section{Introduction}

Screening of various body fluids to detect presence of drugs of abuse is a very useful technique in clinical, forensic and research contexts. Various body fluids and tissues are used for this screening including urine, blood, saliva, sweat, hair and nails etc., with each having their own advantages and disadvantages [1]. However, drugs of abuse have relatively short half life in blood samples. Drugs like benzodiazepines and cocaine containing ester or amide group are prone to degradation by hydrolysis [2]. The instability of drugs in biological samples during transportation and storage possess challenge in the interpretation of results of analysis [3]. Time lapse between the sample collection and actual analysis may protect/hide the suspected drug users from law. Another important aspect is handling the biological samples of the suspected drug users as it is often associated with prevalence of infection with chronic diseases and a risk of transmission of HIV, hepatitis and other blood born viruses [4]. In recent years, dried blood spots have received considerable attention. To reduce the time of sample collection and analysis in a stable matrix and to reduce the handling of infectious diseases the use of dried blood spot (DBS) could be an alternative $[5,6]$.

The use of dried blood spots (Guthrie card) for screening metabolic diseases in large population of neonates was first introduced by Dr. Robert Guthrie in early 1960s [7]. This novel approach for collecting blood has facilitated population screening for newborn and other clinical testing $[8,9]$. Today in USA more than $95 \%$ of newborns are screened for inherited metabolic diseases using DBS [9].

The conventional whole blood, plasma or serum sample collection requires venipuncture while the DBS preparation requires finger or heel prick and spotting on to the filter paper. The detail procedure of sampling \& obtaining DBS is discussed in technique and relevant factors in DBS sampling section. The analysis of blood spots comprises of several challenges, including proper elution of the analyte of interest unlike the conventional whole blood, plasma or serum for screening drugs of abuse.

DBS provide several advantages over conventional whole blood, plasma or serum sample collection $[5,10$, 11]. 1) The method is less invasive (finger or heel prick as compared to conventional venous cannula); 2) It requires simple storage and easier transfer as no freezers or dry ice is required; 3) It is a cost-effective choice for epidemiologic studies; 4) The dried blood matrix stabilizes many analyses and it reduces the infection risk of HIV/AIDS and other infectious pathogens; 5) It also requires small volume.

The filter paper blood collection device has achieved the same level of precision and reproducibility as that of 
standard methods of collecting blood as vaccume tubes or capillary pipettes [5]. DBS application is extended to many fields including epidemiological studies [8], therapeutic drug monitoring [10], toxicokinetics [12] and pharmacokinetics [13]. The use of dried blood spots in screening drug of abuse is the naive area of research. A number of studies have documented the use of dried blood spot for drug analysis. Use of dried blood spots may circumvents various issues like short half life of drugs in blood, time lapse between blood collection and drug analysis, risk of infection and monitoring of therapeutic drugs from a remote place. In this regard it was found worthwhile to provide an overview of the use of dried blood spots in screening drugs of abuse in the literature.

For the sake of clarity of understanding the review has been organized in the following headings: Dried Blood Spot: Sample Collection, Storage \& Transport and Review of Drugs of Abuse Assays with the DBS Method.

\section{Technique and Relevant Factors in DBS Sampling}

\subsection{DBS Sampling Paper}

Schleicher and Scheull 903 (S\&S 903) paper manufactured from $100 \%$ pure cotton linters is recommended for DBS sampling. This paper has been extensively used in newborn screening and for other applications worldwide [14]. The use of S\&S 2992, Whatman grade 1 or 3 has also been reported [5]. In addition, particle retention, pore size and thickness are the properties that determine the loading capacity and spreadability of blood samples onto the paper $[15,16]$. In neonatal screening, the performance of the filter paper blood collection products is monitored by the Newborn Screening Quality Assurance Program (NSQAP) to ensure that the new paper lots are consistent with established guidelines and they perform consistent to the previous lot.

\subsection{Methods for Obtaining Dried Blood Spot}

The dried blood spot is prepared by piercing the skin of either finger or heel or toe with a sterilette and blotted on to high quality filter paper. Other procedures for obtaining DBS are applying blood collected in capillary tubes by dorsal vein puncture onto the preprinted circle of filter paper. The National Committee for Clinical Laboratory Standards (NCCLS) describes in detail the procedure for the collection of blood from heel sticks [17].

\subsection{DBS Specimens Drying, Storage and Transportation}

It is important to dry DBS samples completely before storage or transport because moisture could harm the specimen by inducing bacterial growth or altering the elution [5]. The blood spot specimens should be dried for at least 3 hours over an open nonabsorbent surface at room temperature $\left(15^{\circ} \mathrm{C}-22^{\circ} \mathrm{C}\right)$. The paper should be kept away from direct sunlight and should not be heated, stacked or allowed to touch other surfaces during the drying process. The specimens should be protected against humidity and moisture by packing them in low gas permeable zip closer bags with desiccant packages and humidity indicator cards [17].

DBS samples can be tested immediately. In cases, where samples are not tested immediately DBS samples are stored at ambient temperature or frozen at -20 degree which are stable for several years. In situations where DBS specimens have to be transported to the centralized laboratories, these samples should be packed as described above and can be transported through mail. The zip closer bags containing the packed specimen should be enclosed and sealed in a high quality bond envelope for shipment [18]. Packaging DBS specimens in multiple layers protects mail handlers from accidental exposure and ensures specimen integrity during shipping.

\section{Review of Drugs of Abuse Assays with the DBS Method}

Table 1 provides a list of the available drugs of abuse assays with dried blood spot in the literature [19-27]. For the sake of clarity, data have been presented by individual drug categories (as opposed to the individual studies). In the following sections a brief overview of the same has been discussed.

\subsection{Preparation of Calibrators, Quality Controls and Blank for Dried Blood Assay}

Most of the available studies have reported preparing the calibrators, quality controls and blank for dried blood assay by mixing with washed red blood cells to give an adjusted hematocrit of $55 \% \pm 1 \%$. The hematocrit adjusted blood, spiked with known amount of standard drug concentration of various ranges was spotted. It has been suggested that this process will minimize matrix differences and maximize comparability between calibrators and test samples.

\subsection{DBS Sample Extraction}

Before Testing in the laboratory a disc of dried blood typically $3.0-5.5 \mathrm{~mm}$ in diameter, equivalent to $1-4$ microlitre of serum, was punched either manually or with an automated machine. The punched filter paper disc is either collected in the tube or in the well of the microtitre plate, which is further extracted by liquid solvent extraction technique depending on the drug. The analytes of interest were extracted with gentle shaking or vortexing. 


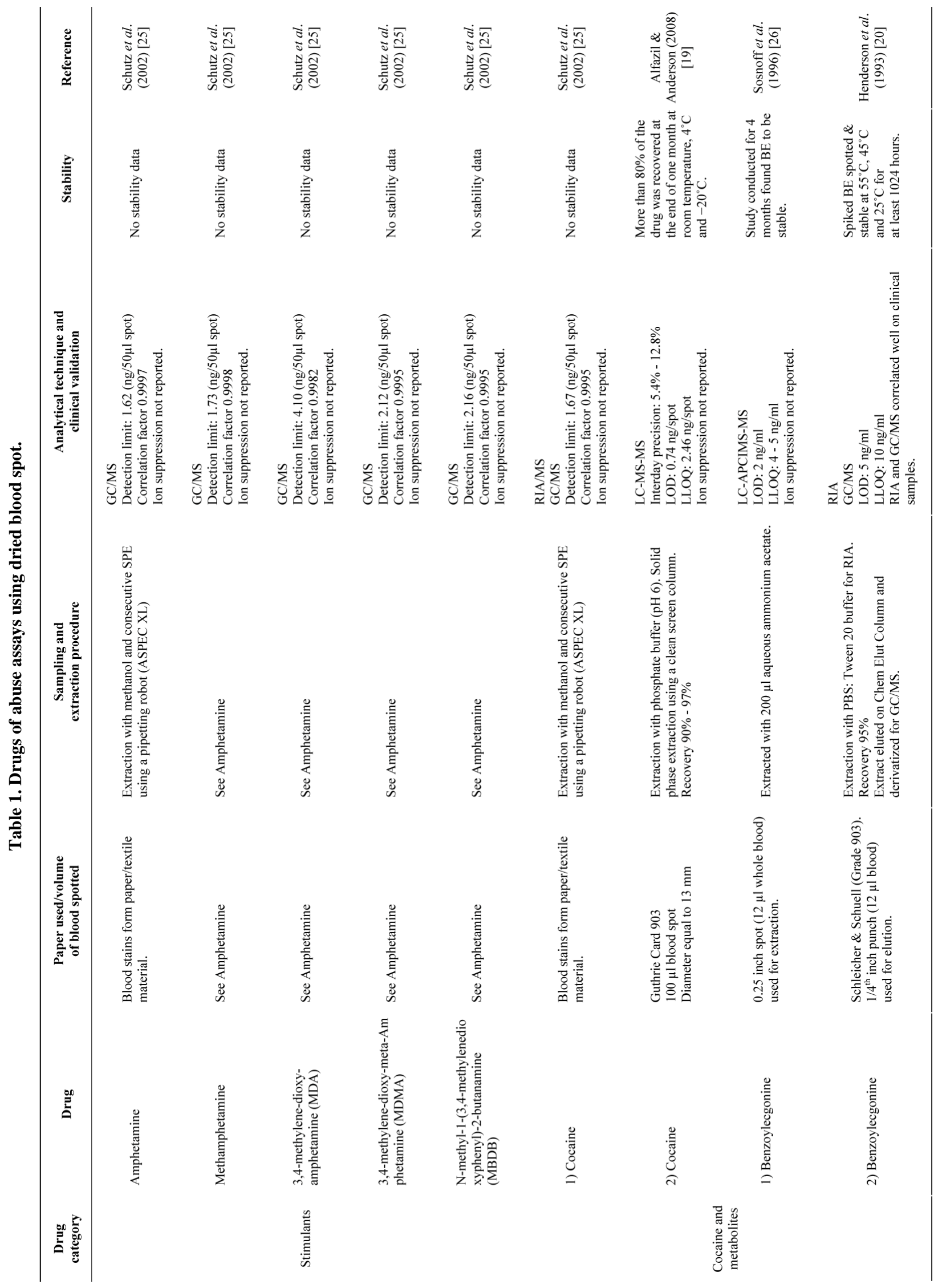




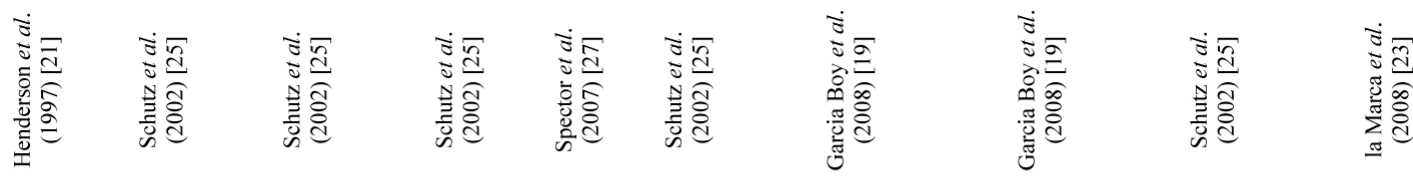

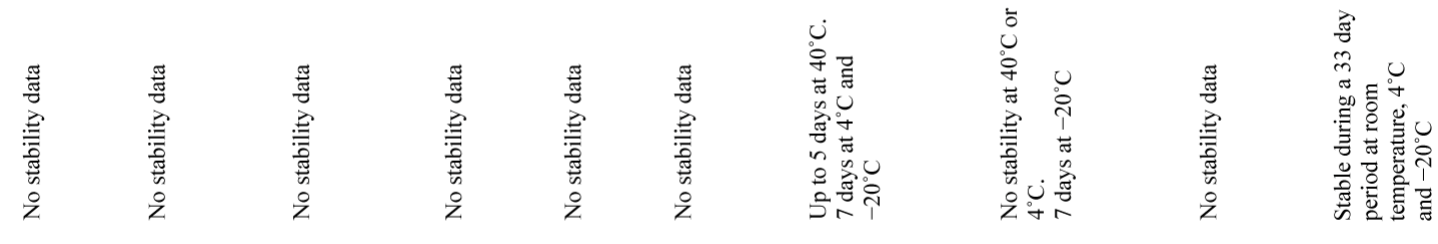

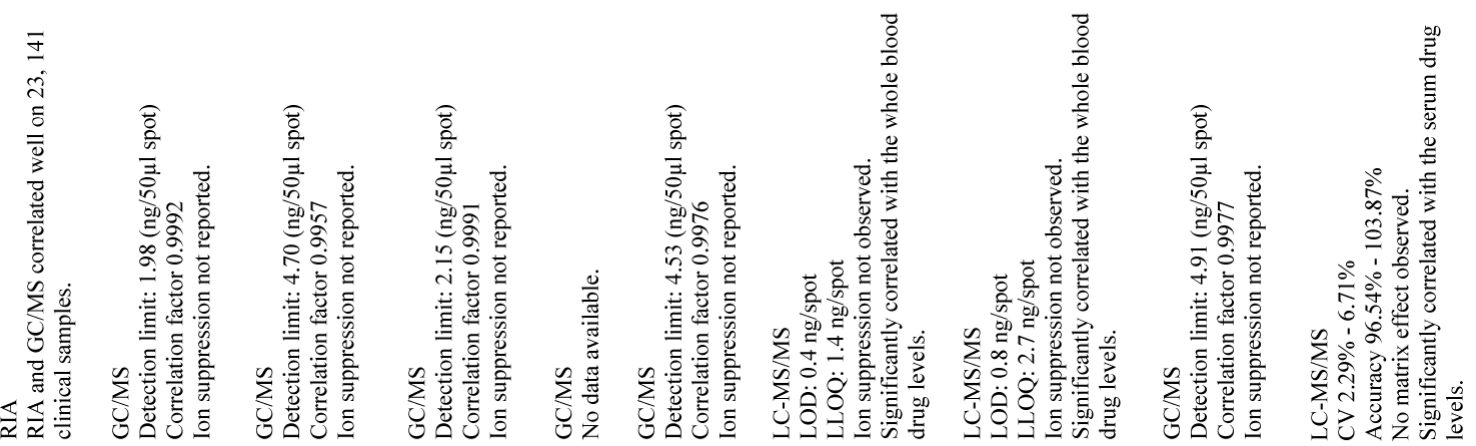

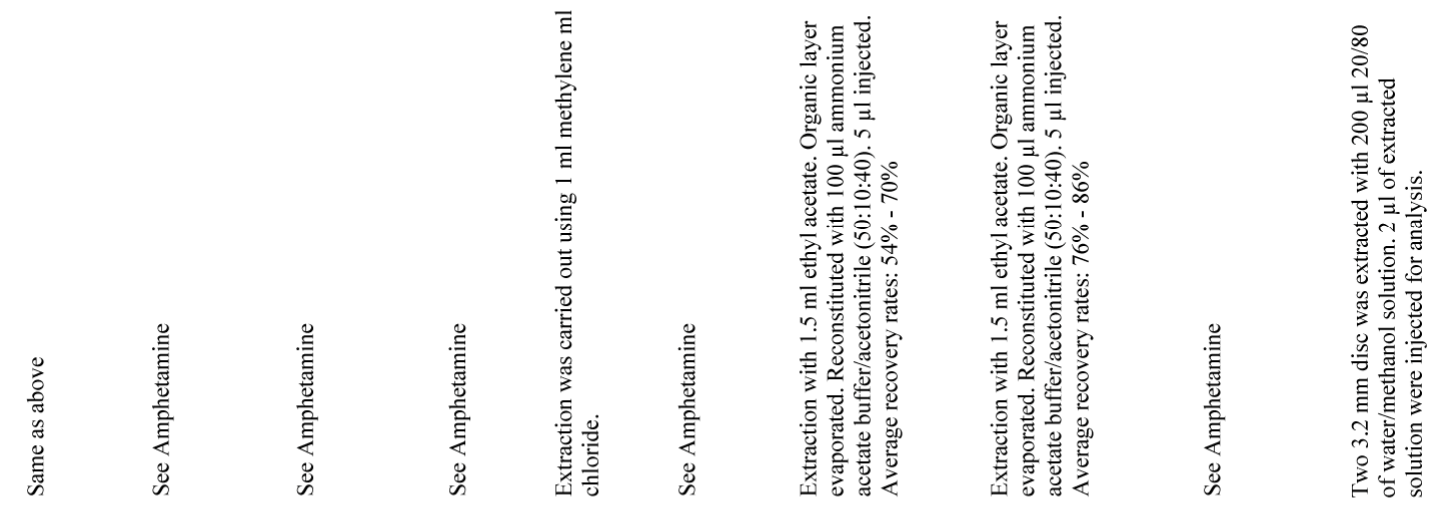

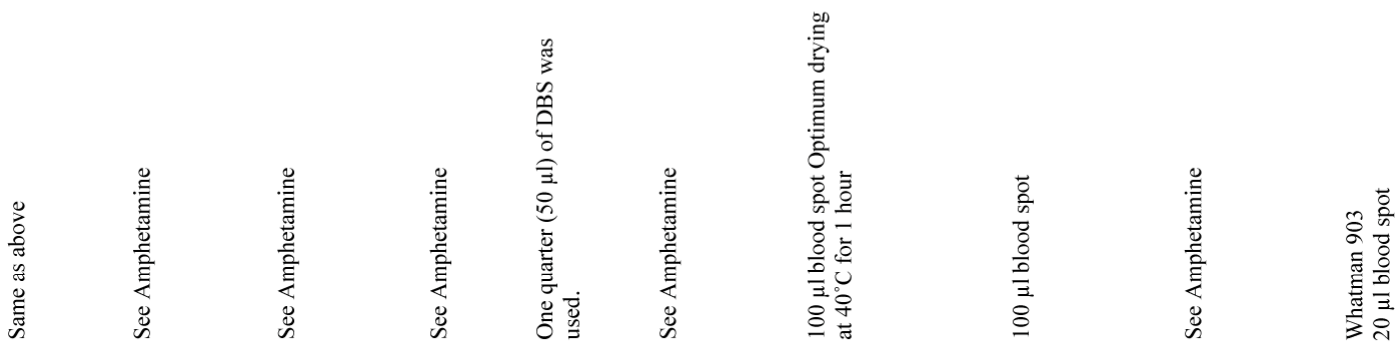

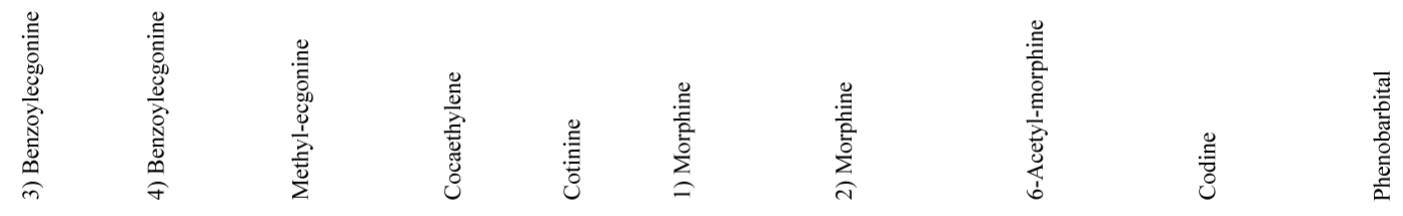




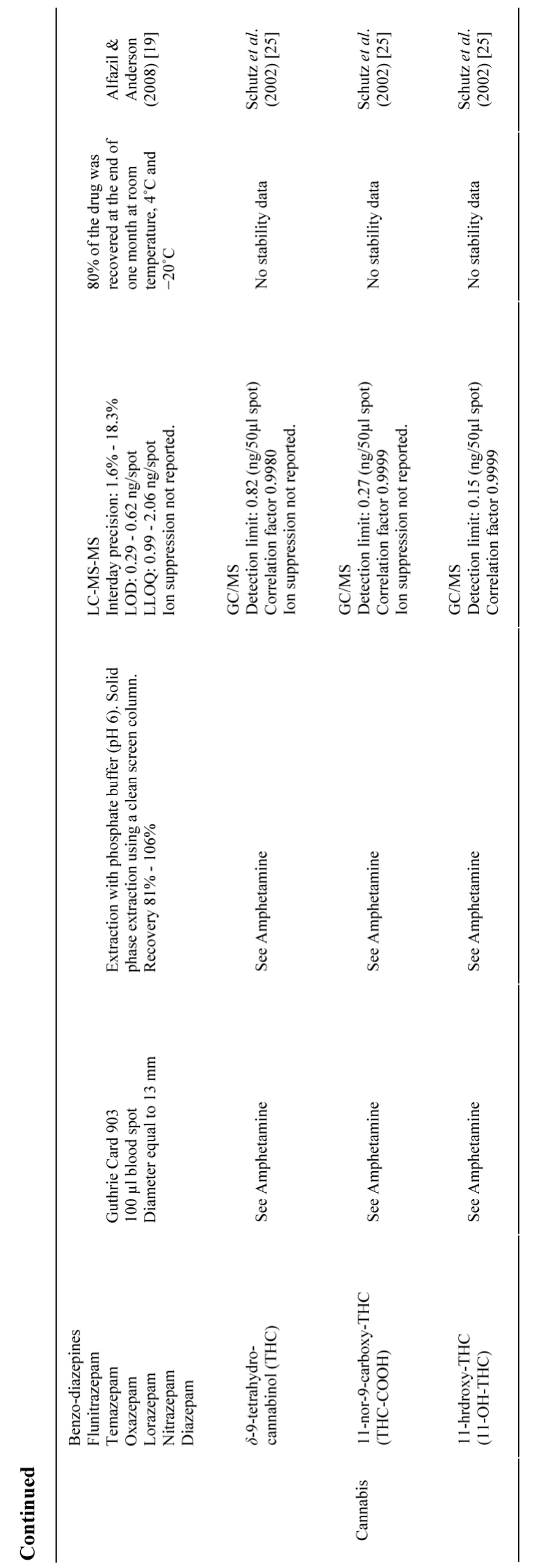


In some assays sonication was done to improve extraction efficiency [19]. After extraction the extract anytes were subjected to either immunological assays or chromatographic techniques as discussed below (Table 1). Moreover, internal standards were added during extraction wherever required in chromatographic techniques for quantitive assays.

\subsection{Derivatization of Drugs}

The chromatographic technique in association with Mass Spectrometry (MS) for reported dried blood assay requires an additional step of derivatization to detect the metabolites of drugs like amphetamines, cocaine, opiates and cannbinoids. The preferred methods reported were silylation or fluoroacetylation. During this process the eluate was nitro evaporated followed by addition of reagents and heated at $100^{\circ} \mathrm{C}$. The cooled derivates were reconstituted and injected for chromatographic analysis.

\subsection{Assay Technique}

The initial studies reported that the determination of drugs of abuse was carried out using immunoassay techniques $[20,21]$. The method was reported to correlate well when compared with gas chromatography/mass spectrometry. However, immunoassays require use of radioactive material and can cross react with endogenous components and non specific binding [22]. Thus, the later studies have reported using either liquid or gas chromatography (LC or GC) associated with mass spectrometry (MS). GC run time is normally long associated with time consuming sample preparation and the necessity of derivation in many drugs for analysis. Recent studies used liquid chromatography associated with tandem mass spectrometry (LC-MS-MS) in the confirmatory analysis [19,23-24].

\subsection{Precision and Accuracy}

Most of the reported literature seems to be properly validated as per standard guidelines from the industry [28] with appropriate intra and inter assay precision and accuracy. The accuracy and precision of a DBS method was evaluated using quality control (QC) samples prepared with varying concentrations (low, mid and high) and analyzed with a nonzero calibration standard in three separate validations run.

\subsection{Matrix Effect and Recovery}

In the reported DBS assays implying MS/MS detection, matrix effect was observed to study the presence of additional matrix. During method development ion suppression/enhancement was reported by post-column infusion of the analyte and internal standard neat solutions while analyzing blank DBS sample extract. Checking of carry over was done by injecting two extracted blank DBS sample sequentially immediately after every 5 - 8 samples [24].

Recovery from extracted dried blood samples was investigated by comparing the results (peak area ratio) obtained from (extracted standard/unextracted standard) $\times$ 100 [24]. Linearity of the assay method, lower limits of detection and quantitation were mentioned in most of the methods descried.

\subsection{Clinical Validation}

Correlation of measured analyte concentration between DBS and venous blood was provided in few studies. A comparison is recommended as part of assay method validation. A good correlation provides confidence in quantative analysis [24]. Phenobarbital levels in plasma correlated well with corresponding DBS when corrected for HCT values [23].

\subsection{Stability}

Only a few studies reported stability of drugs on dried blood specimens [19,23,24]. Dehydration of the samples on the filter paper minimized the hydrolysis of drugs which are liable to this type of degradation and so the stability of the drugs in DBS was enhanced when compared to liquid samples [19]. The various temperature conditions tested for stability were either at room temperature or at $40^{\circ} \mathrm{C}$ for first week and than at $4^{\circ} \mathrm{C}$ and $-20^{\circ} \mathrm{C}$.

\section{Future Perspective}

The use of DBS appears to be promising for determination of drugs of abuse. The advantages of use of DBS over conventional methods of screening drugs of reduce may reduce the time of detection, poses lesser risk of infection from blood born viruses. Also it is helpful in suspected cases of driving under the influence of drugs. Moreover, for large scale epidemiological and field based studies, DBS can minimize the practical hassles associated with blood collection and hence may be cost effective. Furthermore, the method is also suitable for newborn screening (with low sample volume) to identify mothers who were drug users. Drugs and drug metabolites having a shorter half life can be effectively measured using DBS. Additionally, the DBS matrix is found to stabilize the drugs which may help to store the samples for a longer time periods at room temperatures.

However, notwithstanding the obvious advantages of the DBS method it also has certain disadvantages. Small sample volume makes it difficult to perform repeat analysis of the sample. Sensitivity of the assay remains a challenge. The determination of drug metabolites is often difficult as its concentration is usually lower than the 
parent compound. The blood volume, size of the spot and degree of hemolysis are other factors affecting the DBS assay. The hematocrit correlates with drug distribution and the diameter of the blood spot [29]. A low or high hematocrit could thus influence the correct determination and quantification of the desired analyte. The whole blood spot should be used to avoid hematocrit dependent variation. To ensure the ruggedness of the method the quality controls should be prepared with hematocrit values ranging from high to low along with the calibrator standard prepared the same way. Finally, the sensitivity of the DBS method could be improved by using a method requiring low sample volume and lower detection and quantitation limits.

\section{Conclusion}

The DBS sampling has the potential to be a useful technique to detect drugs of abuse. The use of DBS for any drug should be judged against the potential error involved with the method. The quality of sampling paper, standardization and sensitivity of the method are the important factors in the reliability of the DBS method.

\section{REFERENCES}

[1] E. J. Cone, "New Developments in Biological Measures of Drug Prevalence," NIDA Research Monograph, Vol. 167, 1997, pp. 108-129.

[2] O. H. Drummer, "Postmortem Toxicology of Drugs of Abuse," Forensic Science International, Vol. 142, No. 2, 2004, pp. 101-113. doi:10.1016/j.forsciint.2004.02.013

[3] P. Holmgren, H. Druid, A. Holmgren and J. Ahlner, "Stability of Drugs in Stored Post Mortem Femoral Blood and Vitreous Humor," Journal of Forensic Sciences, Vol. 49, No. 4, 2004, pp. 820-825. doi:10.1520/JFS2003433

[4] World Health Organization United Nations Office on Drugs and Crime (UNODC), "Substitution Maintenance Therapy in the Management of Opioid Dependence and HIV/AIDS Prevention," United Nations Organisation, Geneva, 2004.

[5] J. V. Mei, J. R. Alexander, B. W. Adam and W. H. Hannon, "Use of Filter Paper for the Collection and Analysis of Human Whole Blood Specimens," Journal of Nutrition, Vol. 131, No. 5, 2001, pp. 1631S-1636S.

[6] D. Brambilla, C. Jennings, G. Aldrovandi, J. Bremer, A. M. Comeau, S. A. Cassol, R. Dickover, J. B. Jackson, J. Pitt, J. L. Sullivan, A. Butcher, L. Grosso, P. Reichelderfer and S. A. Fiscus, "Multicenter Evaluation of Use of Dried Blood and Plasma Spot Specimens in Quantitative Assays for Human Immunodeficiency Virus RNA: Measurement, Precision and RNA Stability," Journal of Clinical Microbiology, Vol. 41, No. 5, 2003, pp. 1888-1893. doi:10.1128/JCM.41.5.1888-1893.2003

[7] R. Guthrie and A. Susi, "A Simple Method for Detecting Phenylketonuria in Large Populations of Newborn Infants," Pediatrics, Vol. 32, No. 3, 1963, pp. 338-343.
[8] S. P. Parker and W. D. Cubit, "The Use of Dried Blood Samples in Epidemiological Studies," Journal of Clinical Pathology, Vol. 52, 1999, pp. 633-639. doi:10.1136/jep.52.9.633

[9] B. Wilcken and V. Wiley, "Newborn Screening," $P a-$ thology, Vol. 40, No. 2, 2008, pp. 104-115. doi: $10.1080 / 00313020701813743$

[10] P. M. Edelbroek, J. van der Heijden and L. M. Stolk, "Dried Blood Spot Methods in Therapeutic Drug Monitoring: Methods, Assays and Pitfalls," Therapeutic Drug Monitoring, Vol. 31, No. 3, 2009, pp. 327-336. doi:10.1097/FTD.0b013e31819e91ce

[11] T. W. McDade, S. Williams and J. J. Snodgrass, "What a Drop Can Do: Dried Blood Spots as a Minimally Invasive Method for Integrating Biomarkers into Population Based Research," Demography, Vol. 44, No. 4, 2007, pp. 899925. doi:10.1353/dem.2007.0038

[12] M. Barfield, N. Spooner, R. Lad, S. Parry and S. Fowles, "Application of Dried Blood Spots Combined with HPLC-MS/MS for the Quantitation of Acetaminophen in Toxicokinetic Studies," Journal of Chromatography B Analytical Technologies Biomedical and Life Sciences, Vol. 870, No. 1, 2008, pp. 32-37. doi:10.1016/j.jchromb.2008.05.025

[13] N. Spooner, R. Lad and M. Barfield, "Dried Blood Spots as a Sample Collection Technique for the Determination of Pharmacokinetics in Clinical Studies: Consideration for the Validation of a Quantitative Bioanalytical Method," Analytical Chemistry, Vol. 81, No. 4, 2009, pp. 1557-1563. doi:10.1021/ac8022839

[14] W. Li and F. L. S. Tse, "Dried Blood Spot Sampling in Combination with LC-MS/MS for Quantitative Analysis of Small Molecules," Biomedical Chromatography, Vol. 24, No. 1, 2010, pp. 49-65. doi:10.1002/bmc.1367

[15] S. D. O’Broin, B. P. Kelleher and E. Gunter, "Evaluation of Factors Influcing Precision in the Analysis of Samples Taken from Blood Spots on Filter Paper," Clinical and Laboratory Hematology, Vol. 17, No. 2, 1995, pp. 185188.

[16] S. D. O'Broin, "Influence of Hematocrit on Quantitative Analysis of Blood Spots on Filter Paper," Clinical Chemistry, Vol. 39, No. 6, 1993, pp. 1354-1355.

[17] W. H. Hannon, J. Boyle, B. Davin, A. Marsden, E. R. B. McCabe, M. Schwartz, G. Scholl, B. L. Therrell, M. Wolfson and F. Yoder, "Blood Collection on Filter Paper for Neonatal Screening Programs, Approved Standard," Document A4A3, 3rd Edition, National Committee for Clinical laboratory Standards, Wayne, 1997.

[18] R. Knudsen, W. Slazyk and J. Riehmond, "Guidelines for the Shipment of Dried Blood Spots Specimens," Centers for Disease Control and Prevention, Atlanta, 1995. http://www.cdc.gov/od/ohs/biosftydriblood.htm.

[19] A. A. Alfazil and R. A. Anderson, "Stability of Benzodiazepines and Cocaine in Blood Spots Stored on Filter Paper," Journal of Analytical Toxicology, Vol. 32, No. 7, 2008, pp. 511-515.

[20] L. O. Henderson, M. K. Powell, W. H. Hannon, B. B. Miller, M. L. Martin, R. L. Hanzlick, D. Vroon and W. R. Sexson, "Radioimmunoassay Screening of Dried Blood 
Spot Materials for Benzoylecgonine," Journal of Analytical Toxicology, Vol. 17, No. 1, 1993, pp. 42-47.

[21] L. O. Henderson, M. K. Powell, W. H. Hannon, J. T. Bernert, K. A. Pass, P. Fernhoff, C. D. Ferre, L. Martin, E. Franko, R. W. Rochat, M. D. Brantley and E. Sampson, "An Evalution of the Use of Dried Blood Spots from Newborn Screening for Monitoring the Prevalence of Cocaine Use among Childbearing Women," Biochemical and Molecular Medicine, Vol. 61, No. 2, 1997, pp. 143151. doi:10.1006/bmme.1997.2609

[22] T. Wong, C. H. Shackleton, T. R. Covey and G. Ellis, "Identification of the Steroids in Neonatal Plasma that Interfere with 17 Alpha-Hydroxyprogesterone Radioimmunoassay," Clinical Chemistry, Vol. 38, 1992, pp. 387395.

[23] G. La Marca, S. Malvagia, L. Fillipi, F. Luceri, G. Moneti and R. Guerrini, "A New Rapid Micromethod for the Assay of Phenobarbital from Dried Blood Spots by LCTandemmass Spectrometry," Epilepsia, Vol. 50, No. 12, 2009, pp. 2658-2662. doi:10.1111/j.1528-1167.2009.02204.x

[24] R. G. Boy, J. Henseler, R. Mattern and G. Skopp, "Determination of Morphine and 6-Acetylmorphine in Blood with Use of Dried Blood Spots," Therapeutic Drug Monitoring, Vol. 30, No. 6, 2008, pp. 733-739. doi:10.1097/FTD.0b013e31818d9fdb

[25] H. Schutz, J. C. Gotta, M. R. Erdmann and G. Weiler,
"Simultaneous Screening and Detection of Drugs in Small Blood Samples and Blood Stains," Forensic Sciences International, Vol. 126, No. 3, 2002, pp. 191-196. doi:10.1016/S0379-0738(02)00053-1

[26] C. S. Sosnoff, Q. Ann, J. T. Bernert Jr., M. K. Powell, B. B. Miller, L. O. Henderson, W. H. Hannon, P. Fernhoff and E. J. Sampson, "Analysis of Benzoylecgonine in Dried Blood Spots by Liquid Chromatography-Atmospheric Pressure Chemical Ionization Tandem Mass Spectrometry," Journal of Analytical Toxicology, Vol. 20, No. 3, 1996, pp. 179-184.

[27] L. G. Spector, S. S. Hecht, S. Ognjanovic and S. G. Carmella, "Detection of Cotinine in Newborn Dried Blood Spots," Cancer Epidemiology, Biomarkers and Prevention, Vol. 16, 2007, pp. 1902-1905. doi:10.1158/1055-9965.EPI-07-0230

[28] US Department of Health and Human Services, Food and Drug Administration, "Guidance for Industry: Bioanalytical Method Validation," Rockville, 2001. http://www.fda.gov/CDER/GUIDANCE/4252fnl.htm

[29] A. Diamandi, M. J. Khosravi, J. Mistry, V. Martinez and J. G. Aguirre, "Filter Paper Blood Spot Assay of Human Insulin like Growth Factor I (IGF-I) and IGF-Binding Protein-3 and Preliminary Application in the Evaluation of Growth Hormone Status," Journal of Clinical Endocrinology and Metabolism, Vol. 83, No. 7, 1998, pp. 2296-2301. doi:10.1210/jc.83.7.2296 